\title{
Research on pressure induced phase transitions using multiple synchrotron X-ray techniques: from crystalline to non-crystalline samples
}

\author{
Luhong Wang ${ }^{1}$, Arthur Haozhe Liu ${ }^{2}$ \\ ${ }^{1}$ Harbin Institute of Technology, Harbin, People's Republic of China, \\ ${ }^{2}$ HPSTAR, Beijing, People's Republic of China
}

luhong1@hit.edu.cn,haozhe.liu@hpstar.ac.cn

The current state-of-the-art synchrotron x-ray techniques combined with diamond anvil cell (DAC) and large volume press (LVP) techniques make phase transition studies one of most active, burgeoning fields in the high-pressure community. The structural evolution of material under pressure is the long-term active research subject, which in fact strongly depends on the development of corresponding high pressure and synchrotron technologies. The selected research cases for various types of material, such as metallic glasses, melt, and crystalline materials under high pressure conditions, will be presented in this paper. The topics will include pressure-induced polyamorphization in several typical metallic glass systems, pressure-induced potential liquid-liquid in gallium melt, evaluation on novel characteristic method of fractional dimensionality for non-crystalline cases, pressure-induced phase transition consequence trend in metal dioxides at multiple $100 \mathrm{GPa}$ conditions, etc [1-4]. The contributions from the first principal calculations and synergetic effort in synchrotron sources will be discussed based on these scientific cases.

The development of many advanced synchrotron X-ray techniques provided great opportunity for the research under high pressure conditions. Besides the most popular synchrotron X-ray diffraction technique to study the crystalline samples, we could use the high energy X-ray scattering technique combined with the Pair Distribution Function (PDF) method, to study structural evolution of noncrystalline samples in DAC or LVP. In addition, the densities of non-crystalline samples in DAC or LVP could be directly measured by synchrotron X-ray tomographic techniques [5-7]. With instrumental developments in the collection of sparse scattering signals and to an increased flux and coherence of X-ray beams, X-ray photon correlation spectroscopy (XPCS) has recently emerged as a very powerful technique able to follow the evolution of the dynamics at the atomic length scale in crystalline and amorphous materials. Different from the conventional diffraction and scattering methods, which offer the information of average structures over the diffraction volume in sample, the XPCS could uncover the local order from time domain when the coherent beam size is equal to the illuminated sample volume, and exposure time is shorter than the onset time for the speckle dynamics. So the temporal relaxation procedure on the origin of amorphous state to another amorphous state transition process upon compression could be monitored. XPCS experiments under high pressure conditions were performed at room temperature, and results on selected typical metallic glass systems, will be presented by comparing with temperature effect using same XPCS techniques.

[1] Xie, S., Wang, L., Liu, F. Li, X., Bai, L., Prakapenka, V. B., Cai, Z., Mao, H. K., Zhang, S., Liu, H., (2018) J. Phys. Chem. Lett. $9,2388$.

[2] Li, R., Wang, L., Li, L., Yu, T., Zhao, H., Chapman, K. W., Rivers, M. L., Chupas, P. J., Mao, H. K., Liu, H., (2017) Phys. Rev. B, $95,224204$.

[3] Li, L., Luo, Q., Li, R., Zhao, H., Chapman, K. W., Chupas, P. J., Wang, L., Liu, H., (2017) Scientific Reports, 7, 46762.

[4] Li, R., Wang, L., Li, L., Zhao, H., Qu, D., Chapman, K. W., Chupas, P. J., Liu, H. (20160 Physical Review B, 94, 184201.

[5] Li, R., Wang, L., Li, L., Yu, T., Zhao, H., Chapman, K. W., Wang, Y., Rivers, M. L., Chupas, P. J., Mao, H. K., Liu, H., (2017) Scientific Reports, 7, 5666.

[6] Li, R., Li, L., Yu, T., Wang, L., Chen, J., Wang, Y., Cai, Z., Chen, J., Rivers, M. L., Liu, H., (2014) Applied Physics Letters, $105,041906$.

[7] Liu, H., Wang L., Xiao, X., De Carlo, F., Feng, J., Mao, H. K., Hemley, R. J., (2008) PNAS, 105, 13229.

\section{Keywords: High pressure; synchrotron X-ray techniques; XRD; XPCS; tomography}

This work was supported by Natural Science Foundation of China (11374075), Longjiang Scholar, the Fundamental Research Funds for the Central Universities (HIT. BRET1.2010002, HIT. IBRSEM.A.201403). Synchrotron X-ray experiment was performed at Argonne National Laboratory and use of the Advanced Photon Source were supported by the US DoE, Office of Science, Office of Basic Energy Sciences, under contract No. DE-AC02-06CH11357. GSECARS is supported by the National Science Foundation (NSF)-Earth Sciences (EAR-1128799) and DoE-GeoSciences (DE-FG02-94ER14466). Use of the COMPRES-GSECARS gas loading system was supported by COMPRES under NSF Cooperative Agreement EAR 11-57758. 